\title{
Diametrical Elastic Relaxation During Ejection of lactose-cocoa binary tablets.
}

\begin{abstract}
This work investigates the diametrical elastic relaxation of a compacted binary powder mixture of lactose and cocoa. It has been observed that the addition of lactose improved the mechanical strength of the binary tablets at the higher compaction stresses used in this study. The tablets having the maximum tensile strengths have relatively low diametrical elastic relaxations, similar to the pure cocoa tablets. Meanwhile, pure lactose tablets displayed increasing diametrical elastic relaxations as the compaction stress increased.
\end{abstract}

Keyword: Binary tablet, compaction, ejection, elastic relaxation, stored elastic energy, tensile strength 